\title{
BMJ Open Risk factors and determinants of carotid intima-media thickness in children: protocol for a systematic review and meta-analysis
}

\author{
Adina Mihaela Epure, ${ }^{1,2}$ Magali Leyvraz, ${ }^{1}$ Yvan Mivelaz, ${ }^{2}$ Stefano Di Bernardo, ${ }^{2}$ \\ Bruno R da Costa, ${ }^{3}$ Arnaud Chiolero, ${ }^{1,3,4}$ Nicole Sekarski ${ }^{2}$
}

To cite: Epure AM, Leyvraz M, Mivelaz $\mathrm{Y}$, et al. Risk factors and determinants of carotid intimamedia thickness in children: protocol for a systematic review and meta-analysis. BMJ Open 2018;0:e19644. doi:10.1136/ bmjopen-2017-019644

- Prepublication history and additional material for this paper are available online. To view these files, please visit the journal online (http://dx.doi org/10.1136/bmjopen-2017019644).

Received 15 September 2017 Revised 10 April 2018 Accepted 13 April 2018
Check for updates

${ }^{1}$ Institute of Social and Preventive Medicine (IUMSP), Lausanne University Hospital (CHUV), Lausanne, Switzerland ${ }^{2}$ Paediatric Cardiology Unit, Woman-Mother-Child Department, Lausanne University Hospital (CHUV), Lausanne, Switzerland ${ }^{3}$ Institute of Primary Health Care (BIHAM), University of Bern,

Bern, Switzerland

${ }^{4}$ Department of Epidemiology, Biostatistics and Occupational Health, McGill University, Montreal, Canada

Correspondence to

Adina Mihaela Epure;

adina-mihaela.epure@chuv.ch

\section{ABSTRACT}

Introduction Carotid intima-media thickness (CIMT) is a surrogate marker of atherosclerosis that is measured in adults and children to better understand the natural history of cardiovascular disease (CVD). In adults, CIMT is predictive of myocardial infarction and stroke. In children and adolescents, CIMT is used to assess vascular changes in the presence of CVD risk factors (obesity, hypertension, smoking, etc) or clinical conditions associated with a high risk for premature CVD. However, there is no comprehensive overview, in a life-course epidemiology perspective, of the risk factors and determinants of CIMT in children. It is also important to evaluate between-study differences in CIMT measurement methods and take them into consideration when drawing conclusions. Our objective is to systematically review the evidence on the relationship between CIMT and prenatal and postnatal exposures or interventions in children, as well as documenting and discussing the CIMT measurement methods.

Methods and analysis Systematic searches of the Medical Literature Analysis and Retrieval System Online (MEDLINE), Excerpta Medica (EMBASE)and Central Register of Controlled Trials (CENTRAL) databases will be conducted. The reference lists and other literatures sources will be browsed. Observational and experimental studies in children from birth up to 18 years will be included. Prenatal and postnatal exposures or interventions assessed in relationship with CIMT will be considered for inclusion. Examples might include gestational age, obesity, hypertension, tobacco exposure, specific at-risk conditions (chronic kidney disease, diabetes, etc) or statin treatment. The outcome will be CIMT assessed by ultrasonography. The setting, scanning and measurement methods for each included study will be described in detail. Results will be synthesised descriptively and, if appropriate, will be pooled across studies to perform metaanalyses. Separate meta-analyses for each exposure or intervention type will be conducted.

Ethics and dissemination This systematic review will be published in a peer-reviewed journal. A report will be prepared for clinicians and other healthcare decisionmakers.

PROSPERO registration number CRD42017075169.
Strengths and limitations of this study

- A critical review of the methodology of the studies and the carotid intima-media thickness measurement methods will be included.

- Most evidence for this systematic review is expected to derive from observational studies, thus limiting our ability to assess causal relationships, yet facilitating the generalisability of findings across different populations and settings.

- The review methods were carefully planned according to current guidelines and prospectively registered with the International Prospective Register of Systematic Reviews (PROSPERO) in order to minimise risk of bias related to study design and conduct, or reporting of results in the completed review.

\section{INTRODUCTION \\ Rationale}

Ultrasound carotid intima-media thickness (CIMT) is a non-invasive technique that is used in adult and paediatric populations for the assessment of subclinical cardiovascular disease (CVD) and as a surrogate end point for treatment efficacy in research settings. ${ }^{1-4}$ Ultrasound CIMT correlates well with its histological measurement ${ }^{5}$ and coronary atherosclerosis. ${ }^{6}$ It has been argued, however, that elevated CIMT is not always equivalent to subclinical atherosclerosis. At lower degrees of thickness and at early ages, it may represent hypertrophy of the intimal and medial layers in the absence of true atherosclerotic lesions. ${ }^{78}$ Nevertheless, increased CIMT as an adaptive response to blood flow, intraluminal pressure or other biological factors relates to the known pathophysiology of atherosclerosis. ${ }^{9} 10$

In adults, CIMT is associated with traditional CVD risk factors ${ }^{11}$ and is predictive of heart attack and stroke. ${ }^{12-17}$ Longitudinal studies showed that elevated CIMT in 
adulthood is linked to risk factors in childhood, ${ }^{18}$ such as obesity, high serum cholesterol and insulin levels. ${ }^{19-21}$ In children, several studies assessed CIMT and its relation with risk factors such as obesity, ${ }^{22}{ }^{23}$ dyslipidaemia, ${ }^{324}$ elevated blood pressure ${ }^{25} 26$ or smoking. ${ }^{27}$ CIMT was also used to assess CVD risk in paediatric patients with clinical conditions that are associated with accelerated atherosclerosis, for instance, diabetes or chronic kidney disease. ${ }^{28-34}$ Clinical trials in children and adolescents showed a decrease in CIMT following interventions to control risk factors. ${ }^{35}$ Furthermore, there is growing evidence that prenatal factors are also determinants of cardiovascular health across the life course and may have an effect on CIMT. $^{37}{ }^{38}$ These data provide support for CIMT as a marker of vascular remodelling in youth. However, it remains to be determined among which children CIMT is increased and if it is clinically relevant to measure it. ${ }^{39}$ Further, a comprehensive overview, in a life-course epidemiology perspective ${ }^{40}$ of the prenatal and postnatal factors associated with CIMT in children is lacking.

The heterogeneity in CIMT measurement methods requires careful consideration as several methodological aspects related to the site of measurement, the edge detection approach and ultrasound settings, or the training level of the operators influence the quality and interpretation of results. ${ }^{91-43}$ Standardised equipment and imaging protocols, as well as sonographers' high expertise, are essential for CIMT acquisition and analysis. ${ }^{42}$ Also, the technique can be challenging, especially in young children, depending on the patient's compliance and anatomic particularities. ${ }^{39}$ Still, our research group proved that CIMT assessment is feasible and reproducible in non-sedated infants. ${ }^{45}$ Due to differences in CIMT measurement technique, the between-study comparability is commonly considered to be limited. Nevertheless, a formal evaluation of the CIMT measurement methods in children is needed for further clarification of this limitation.

\section{Objectives}

Therefore, our objective is to systematically review the evidence on the relationship between CIMT and prenatal and postnatal exposures or interventions in children from birth up to 18 years, as well as documenting and discussing the CIMT measurement methods.

\section{METHODS AND ANALYSIS}

The protocol has been developed according to the Preferred Reporting Items for Systematic Reviews and Meta-Analyses (PRISMA) statements, ${ }^{46-48}$ the Meta-analysis of Observational Studies in Epidemiology (MOOSE) guidelines ${ }^{49}$ and following methods outlined in the Cochrane Handbook for Systematic Reviews of Interventions. ${ }^{50}$ This systematic review has been registered with the International Prospective Register of Systematic Reviews (registration number CRD42017075169).

\section{Eligibility criteria}

Study designs

Observational and experimental primary research studies will be eligible. The following study designs will be considered: cohort, case-control and cross-sectional studies; randomised and non-randomised, controlled and non-controlled trials; quasi-experimental studies. Case reports, case series, opinion papers, letters to the editor, comments, conference proceedings, policy papers, reviews and meta-analyses, study protocols without baseline data and animal studies will be excluded.

\section{Participants}

We will include studies in children from birth up to 18 years. Studies with both children and adults will be included if the data for children can be extracted separately. Both apparently healthy children and subjects with clinical conditions will be included.

\section{Exposures/interventions}

There will be no restrictions regarding the types of exposures or interventions considered in the primary studies. Prenatal and postnatal exposures, at the individual or environmental level, will be considered for inclusion. Examples might include gestational age, birth weight, maternal gestational diabetes, traditional CVD risk factors (blood pressure, blood lipids, etc), specific at-risk clinical conditions (chronic kidney disease, diabetes mellitus, familial hypercholesterolemia, HIV infection, chronic inflammatory disease, etc) or tobacco exposure. For experimental studies, we will consider both pharmacological and non-pharmacological interventions (eg, interventions to promote physical activity or smoking cessation, treatments with antihypertensive or lipid-lowering drugs).

\section{Comparators}

Studies assessing specific clinical conditions in relationship with CIMT will be included provided they use a control group without the clinical condition of interest.

\section{Outcome measures}

The outcome will be the intima-media thickness of the carotid artery measured by ultrasonography.

\section{Time frame}

There will be no restriction by duration of intervention or by length of follow-up.

\section{Setting}

There will be no restriction by type of setting.

Language

Studies in English and French will be included.

\section{Search strategy}

Systematic searches will be conducted in the following databases: (1) Medical Literature Analysis and Retrieval System Online (MEDLINE) from 1946 onwards (PubMed interface); (2) Excerpta Medica database (EMBASE) from 1947 to present (via http://embase. 
Box 1 Search strategy for Medical Literature Analysis and Retrieval System Online (MEDLINE)

1. baby[tiab] $\mathrm{OR}$ babies[tiab] $\mathrm{OR}$ preterm*[tiab] $\mathrm{OR}$ pre-term*[tiab] $\mathrm{OR}$ prematur*[tiab] OR newborn*[tiab] OR infan*[tiab] OR infant[mh] OR toddler*[tiab] OR kindergart*[tiab] OR kid[tiab] OR kids[tiab] OR boy*[tiab] OR girl*[tiab] OR preschool*[tiab] OR pre-school*[tiab] OR child*[tiab] OR child[mh] OR school*[tiab] OR preteen*[tiab] OR prepube*[tiab] OR preadolescen*[tiab] OR highschool*[tiab] OR high-school*[tiab] OR student*[tiab] OR adolescen*[tiab] OR adolescent[mh] OR teen*[tiab] OR pube*[tiab] OR youngster*[tiab] OR youth*[tiab] OR pediatric*[tiab] OR paediatric*[tiab] OR peadiatric*[tiab] OR pediatrics[mh] OR neonat* ${ }^{\star}$ tiab] $O R$ perinat* ${ }^{\star}[$ tiab] $O R$ offspring[tiab] OR descendant*[tiab]

2. "intima media thickness" [tiab] OR "intima media thickening" [tiab] OR "intimal medial thickness" [tiab] OR "intimal medial thickening" [tiab] OR "intimal media thickness"[tiab] OR "intimal media thickening"[tiab] OR "intima medial thickness"[tiab] OR "intima medial thickening"[tiab] OR "intima media complex"[tiab] OR "intimal medial complex" [tiab] OR "intimal media complex"[tiab] OR "intimamedia thickness"[tiab] OR "wall thickness" [tiab] OR "wall thickening" [tiab] OR "arterial thickness" [tiab] OR "artery thickness" [tiab] OR "artery wall thickness"[tiab] OR "arterial wall thickness" [tiab] OR "intimal thickening" [tiab] OR "tunica intima/diagnostic imaging" [mh] OR "tunica media/diagnostic imaging"[mh]

3. carotid[tiab] OR "arteria carotis"[tiab] OR "carotid arteries"[mh] OR "carotid artery diseases" [mh:noexp]

4. atherosclero*[tiab] $\mathrm{OR}$ atherosclerosis[mh] $\mathrm{OR}$ arteriosclero*[tiab] OR arteriosclerosis[mh] OR "end organ damage" [tiab] OR "target organ damage" [tiab] OR "cardiovascular diseases" [mh:noexp]

5. ultrasound[tiab] OR echograph*[tiab] OR ultrasonograph*[tiab] OR sonograph$^{*}[$ tiab] $O R$ ultrasonography[mh]

6. \#2 AND \#3

7. \#3 AND \#4 AND \#5

8. "carotid intima-media thickness" [mh] OR "carotid arteries/diagnostic imaging" [mh]

9. \#6 OR \#7 OR \#8

10. \#1 AND \#9

11. \#10 NOT (animals[mh] NOT humans[mh])

mh, Medical Subject Headings terms; tiab, title/abstract.

com); and (3) Cochrane Central Register of Controlled Trials (CENTRAL) from 1947 onwards (Wiley interface). The search strategy structures for these databases were reviewed and refined by two experienced librarians. They were constructed to include the two main concepts of this systematic review: (1) children and adolescents and (2) CIMT. The search strategy for MEDLINE (Box 1) was created first and then adapted for the other two databases (supplementary appendix 1 and 2 in the online supplementary material file). The reference lists of the retrieved articles and other reviews in the field will be browsed to identify further studies of interest. Supplementary searches will be conducted on Web of Science and Google Scholar. To identify unpublished or ongoing studies, we will search the following registers: the ClinicalTrials.gov (http:/ /www.clinicaltrials.gov), the EU Clinical Trials Register (http://www.clinicaltrialsregister.eu) and the International Clinical Trials Registry Platform (http://www.isrctn.com). The principal investigators of completed studies found on these trial registers will be contacted, via email, to access unpublished data.

\section{Study selection}

An initial evaluation of the collection of articles will be performed to eliminate duplicate publications. Studies will be assessed for inclusion against prespecified criteria independently and in parallel by two reviewers (AME and ML). This process will be conducted in two stages, initially on the basis of titles and abstracts and then by reviewing the full text of the articles retained in the first step. Any disagreements between the two reviewers will be solved by discussion. If consensus is not reached, arbitration by a third reviewer (AC) will be required. The decisions made for each article will be recorded. The systematic reviews software Covidence (Veritas Health Innovation, Melbourne, Australia; http://www.covidence.org) will be used to manage the study selection process.

\section{Data extraction}

Data will be extracted independently by two reviewers (AME and ML) using an electronic database created in Microsoft Office Excel 2007. Disagreements will be resolved by discussion or, if necessary, with the arbitration of a third reviewer (AC). Drop-down lists will be created whenever appropriate to minimise errors. Calibration exercises will be conducted before this review stage to enhance consistency between assessors. Two members of the review team (ML and $\mathrm{AC}$ ) have previous experience in study quality appraisal and data collection for systematic reviews. ${ }^{51-54}$

The following data items will be extracted:

1. Study identification: authors, publication year, journal, funding or sponsorship.

2. Study characteristics: study type (observational or experimental), study design, country, sampling procedure, sample size, study setting, study duration or period of follow-up, participation rates.

3. Study subjects characteristics: inclusion and exclusion criteria, age, sex, ethnicity, anthropometric measures; we will also extract data on medication/co-interventions and comorbidities in children with clinical conditions.

4. Exposures or interventions:

- Exposure type and its characteristics (definition and cut-offs, measurement method, follow-up period/ duration of exposure).

- Intervention type and its characteristics (dosage/intensity, frequency, duration, personnel who deliver it, etc).

5. Outcome:

- CIMT definition and additional features of the CIMT measurement method (Box 2), number of measurements.

6. Potential confounding and effect modifiers:

- Confounding factors and effect modifiers considered in the primary studies, depending on the expo- 
Box 2 Carotid intima-media thickness (CIMT)

characteristics

Equipment

- Ultrasound device type

- Ultrasound settings: imaging technique (eg, B-mode, M-mode), transducer (array, frequency)

- Cardiac cycle tracking method (eg, electrocardiography, other)

- Data storage facilities

Image acquisition and analysis

- Side (right, left, combination), segment (common carotid artery, internal carotid artery, carotid bifurcation, combination), wall (far-wall, near-wall, combination), angle of insonation

- Atherosclerotic plaque (inclusion or exclusion of plaque, definition of plaque)

- Type of measurement (eg, manual, semiautomatic or automatic)

- Timing during the cardiac cycle (eg, irrespective of the cardiac cycle, end-diastole, other)

- Segmental calculation (ie, mean or maximum CIMT)

Quality control procedures

- Use of a predefined standardised imaging protocol, training level of operators, type and number of operators (sonographers, readers)

- Assessment of reproducibility of measurements (ie, intraoperator and/or interoperator variability indices)

- Any other quality control measures taken (eg, phantom scans, maintenance routine of the equipment)

sure or intervention of interest, will be documented and taken into account when performing data analysis (eg, gestational age, birth weight, blood pressure, blood lipids, blood glucose, tobacco exposure, family history of CVD, physical activity or sedentary behaviour).

- Adjusted and unadjusted measures of effect or association will be recorded.

We will collate information provided in multiple reports of the same study if they aim to assess the same exposure/ intervention. If their aim is to assess different exposures/ interventions, multiple reports of the same study will be considered separately in this systematic review. When CIMT measurements are available at several time points, the time point closest to the end of the intervention or the follow-up period will be selected for data extraction. When essential information is missing from the published reports, we will contact the authors of the original studies by email to access missing data. A maximum of three email attempts per study will be undertaken.

\section{Study quality}

Quality appraisal will be performed independently by two reviewers (AME and ML). Any disagreements will be solved by discussion or, if necessary, with the arbitration of a third reviewer (AC). Quality assessment of experimental studies will be conducted in line with the Cochrane collaboration's risk of bias tool. ${ }^{50}$ Observational studies will be evaluated using the Newcastle-Ottawa Scale for non-randomised studies. ${ }^{55}$

The reliability of the CIMT measurement methods used in primary studies will be evaluated following a predefined tool developed for the current review based on existing standards, ${ }^{93941425657}$ and with input from the review team members with expertise in CIMT measurement (YM, SDB, NS). The following three domains will be appraised: (1) site of measurement, (2) image analysis methods and (3) assessment of reproducibility. The complete tool and the algorithm of judgement are presented in the online supplementary appendix 3 .

\section{Data analysis}

The following software programs will be used to carry out statistical analyses: RStudio (V.0.99.473), Stata (V.14.1) and RevMan (V.5.3) (Copenhagen: The Nordic Cochrane Centre, 2014).

\section{Measures of effect or association}

For experimental studies, the measure of effect for continuous outcomes will be the difference in means between the intervention and the control groups. For categorical outcomes, the measure of effect will be the OR or the relative risk. For observational studies, the measure of association for continuous outcomes will be the correlation or regression coefficient, or the difference in means between the exposure and the control groups. For categorical outcomes, the measure of association will be the $\mathrm{OR}$ at different exposure levels.

\section{Descriptive analyses}

The characteristics of the study population and details about the CIMT measurement methods reported in each study will be presented in text and tables. We will also perform a descriptive analysis of studies: (1) not providing sufficient data to be included in the meta-analyses and (2) reporting results in a format that cannot be converted to a standard metric and/or common effect size measure.

\section{Meta-analyses}

If possible, we will pool results across studies for each type of exposure or intervention. This will be conducted separately for observational and experimental studies. Meta-analyses will be computed using random-effects models as heterogeneity is expected.$^{58}$ Results will be presented graphically through forest plots. If studies are not sufficiently homogenous to be combined in a quantitative synthesis, their results will be presented in a narrative format or in graphical displays having the pooled estimate suppressed. ${ }^{50}$ The comparability of studies will be judged based on the CIMT definition and the clinical characteristics of the studied population.

\section{Assessment of heterogeneity}

Statistical heterogeneity will be evaluated using the Cochran's $Q$ test and will be quantified using the $\mathrm{I}^{2}$ method. ${ }^{50}$ If sufficient studies are available, the sources of variability will be investigated by means of subgroup analyses accompanied by interaction tests and meta-regression based on the following variables: 
- Study design, study setting, duration of intervention or duration of exposure/follow-up.

- Sample characteristics: sample size, age, sex.

- Properties of the CIMT measurement method: far-wall versus near-wall or combination of walls, one side versus both sides, manual versus semiautomatic/ automatic measurements, transducer frequency.

Sensitivity analyses will be carried out by (1) excluding relatively small studies, (2) excluding studies of low quality and (3) restricting analyses to studies having the end point defined as mean CIMT.

\section{Assessment of publication bias}

Publication bias will be assessed by visual inspection of funnel plots, and, if sufficient studies will be available, funnel plot asymmetry will be examined using the Egger's test. $^{50} 59$

\section{Assessment of strength of evidence}

The Grading of Recommendations, Assessment, Development and Evaluation (GRADE) framework will be applied in order to assess the strength of the body of evidence for this systematic review. ${ }^{60}$

\section{Patient and public involvement}

This is a protocol for a systematic review and there was no patient or public involvement in framing the research objective or study design.

Acknowledgements The authors thank Cécile Jaques from the Medical Library, Research and Education Department, Lausanne University Hospital (CHUV), Lausanne, Switzerland, and Thomas Brauchli from the Institute of Social and Preventive Medicine (IUMSP), Lausanne University Hospital (CHUV), Lausanne, Switzerland, for their help in reviewing and refining the search strategies for this systematic review.

Contributors AME, AC and NS designed the research protocol for this systematic review. YM, SDB and NS revised the CIMT Measurement Method Reliability Tool. $B R d C$ revised the planned statistical analyses. AME and $A C$ drafted this manuscript. ML, YM, SDB, BRdC and NS critically reviewed the manuscript and provided commentaries. All authors agreed on the final version of the protocol.

Funding This systematic review is funded by the Swiss National Science Foundation (SNSF) (funding reference number 32003B-163240).

Disclaimer The funder will have no involvement in any phase of this study, that is, protocol design and implementation, data analysis and interpretation, or dissemination of results. In the event of protocol amendments, they will be recorded and a rationale for the changes will be provided.

Competing interests None declared.

Patient consent Not required.

Provenance and peer review Not commissioned; externally peer reviewed.

Data sharing statement It is the intention of the authors that once the results and conclusions of this systematic review have been published, the dataset supporting the analyses to be made available in the DATA@IUMSP repository, https://data. iumsp.ch.

Open Access This is an Open Access article distributed in accordance with the Creative Commons Attribution Non Commercial (CC BY-NC 4.0) license, which permits others to distribute, remix, adapt, build upon this work non-commercially, and license their derivative works on different terms, provided the original work is properly cited and the use is non-commercial. See: http://creativecommons.org/ licenses/by-nc/4.0/

(c) Article author(s) (or their employer(s) unless otherwise stated in the text of the article) 2018. All rights reserved. No commercial use is permitted unless otherwise expressly granted.

\section{REFERENCES}

1. van den Oord SC, Sijbrands EJ, ten Kate GL, et al. Carotid intimamedia thickness for cardiovascular risk assessment: systematic review and meta-analysis. Atherosclerosis 2013;228:1-11.

2. O'Leary DH, Bots ML. Imaging of atherosclerosis: carotid intimamedia thickness. Eur Heart J 2010;31:1682-9.

3. Narverud I, Retterstøl K, Iversen PO, et al. Markers of atherosclerotic development in children with familial hypercholesterolemia: a literature review. Atherosclerosis 2014;235:299-309.

4. Groner JA, Joshi M, Bauer JA. Pediatric precursors of adult cardiovascular disease: noninvasive assessment of early vascular changes in children and adolescents. Pediatrics 2006;118:1683-91.

5. Wong M, Edelstein J, Wollman J, et al. Ultrasonic-pathological comparison of the human arterial wall. Verification of intima-media thickness. Arterioscler Thromb 1993;13:482-6.

6. Amato M, Montorsi P, Ravani A, et al. Carotid intima-media thickness by B-mode ultrasound as surrogate of coronary atherosclerosis: correlation with quantitative coronary angiography and coronary intravascular ultrasound findings. Eur Heart $J$ 2007;28:2094-101.

7. Bots ML, Hofman A, Grobbee DE. Increased common carotid intima-media thickness. Adaptive response or a reflection of atherosclerosis? Findings from the Rotterdam Study. Stroke 1997;28:2442-7.

8. Finn AV, Kolodgie FD, Virmani R. Correlation between carotid intimal/ medial thickness and atherosclerosis: a point of view from pathology. Arterioscler Thromb Vasc Biol 2010;30:177-81.

9. Stein JH, Korcarz CE, Hurst RT, et al. Use of carotid ultrasound to identify subclinical vascular disease and evaluate cardiovascular disease risk: a consensus statement from the American Society of Echocardiography Carotid Intima-Media Thickness Task Force. Endorsed by the Society for Vascular Medicine. J Am Soc Echocardiogr 2008;21:93-111. quiz 89-90.

10. Kwak BR, Bäck M, Bochaton-Piallat ML, et al. Biomechanical factors in atherosclerosis: mechanisms and clinical implications. Eur Heart $J$ 2014;35:3013-20.

11. Vos LE, Oren A, Uiterwaal C, et al. Adolescent blood pressure and blood pressure tracking into young adulthood are related to subclinical atherosclerosis: the Atherosclerosis Risk in Young Adults (ARYA) study. Am J Hypertens 2003;16:549-55.

12. Hodis HN, Mack WJ, LaBree L, et al. The role of carotid arterial intima-media thickness in predicting clinical coronary events. Ann Intern Med 1998;128:262-9.

13. O'Leary DH, Polak JF, Kronmal RA, et al. Carotid-artery intima and media thickness as a risk factor for myocardial infarction and stroke in older adults. Cardiovascular Health Study Collaborative Research Group. N Engl J Med 1999;340:14-22.

14. Salonen JT, Salonen R. Ultrasonographically assessed carotid morphology and the risk of coronary heart disease. Arterioscler Thromb 1991;11:1245-9.

15. Simons PC, Algra A, Bots ML, et al. Common carotid intima-media thickness and arterial stiffness: indicators of cardiovascular risk in high-risk patients. The SMART Study (Second Manifestations of ARTerial disease). Circulation 1999;100:951-7.

16. Chambless LE, Heiss G, Folsom AR, et al. Association of coronary heart disease incidence with carotid arterial wall thickness and major risk factors: the Atherosclerosis Risk in Communities (ARIC) Study, 1987-1993. Am J Epidemiol 1997;146:483-94.

17. Lorenz MW, Markus HS, Bots ML, et al. Prediction of clinical cardiovascular events with carotid intima-media thickness: a systematic review and meta-analysis. Circulation 2007;115:459-67.

18. Juonala M, Magnussen CG, Venn A, et al. Influence of age on associations between childhood risk factors and carotid intimamedia thickness in adulthood: the Cardiovascular Risk in Young Finns Study, the Childhood Determinants of Adult Health Study, the Bogalusa Heart Study, and the Muscatine Study for the International Childhood Cardiovascular Cohort (i3C) Consortium. Circulation 2010;122:2514-20.

19. Koskinen J, Kähönen M, Viikari JS, et al. Conventional cardiovascular risk factors and metabolic syndrome in predicting carotid intimamedia thickness progression in young adults: the cardiovascular risk in young Finns study. Circulation 2009;120:229-36.

20. Davis PH, Dawson JD, Riley WA, et al. Carotid intimal-medial thickness is related to cardiovascular risk factors measured from childhood through middle age: The Muscatine Study. Circulation 2001;104:2815-9.

21. Li S, Chen W, Srinivasan SR, et al. Childhood cardiovascular risk factors and carotid vascular changes in adulthood: the Bogalusa Heart Study. JAMA 2003;290:2271-6.

22. Meyer AA, Kundt G, Steiner M, et al. Impaired flow-mediated vasodilation, carotid artery intima-media thickening, and elevated 
endothelial plasma markers in obese children: the impact of cardiovascular risk factors. Pediatrics 2006;117:1560-7.

23. Woo KS, Chook P, Yu CW, Cw Y, et al. Overweight in children is associated with arterial endothelial dysfunction and intima-media thickening. Int J Obes Relat Metab Disord 2004;28:852-7.

24. Wiegman A, de Groot E, Hutten BA, et al. Arterial intima-media thickness in children heterozygous for familial hypercholesterolaemia. Lancet 2004;363:369-70.

25. Lande MB, Carson NL, Roy J, et al. Effects of childhood primary hypertension on carotid intima media thickness: a matched controlled study. Hypertension 2006;48:40-4.

26. Sorof JM, Alexandrov AV, Cardwell G, et al. Carotid artery intimalmedial thickness and left ventricular hypertrophy in children with elevated blood pressure. Pediatrics 2003;111:61-6.

27. Dratva J, Probst-Hensch N, Schmidt-Trucksäss A, et al. Atherogenesis in youth--early consequence of adolescent smoking. Atherosclerosis 2013;230:304-9.

28. Järvisalo MJ, Jartti L, Näntö-Salonen K, et al. Increased aortic intima-media thickness: a marker of preclinical atherosclerosis in high-risk children. Circulation 2001;104:2943-7.

29. Noto N, Okada T, Yamasuge M, et al. Noninvasive assessment of the early progression of atherosclerosis in adolescents with Kawasaki disease and coronary artery lesions. Pediatrics 2001;107:1095-9.

30. Vriend JW, de Groot E, de Waal TT, et al. Increased carotid and femoral intima-media thickness in patients after repair of aortic coarctation: influence of early repair. Am Heart J 2006;151:242-7.

31. Mitsnefes MM, Kimball TR, Witt SA, et al. Abnormal carotid artery structure and function in children and adolescents with successfu renal transplantation. Circulation 2004;110:97-101.

32. Singh TP, Groehn H, Kazmers A. Vascular function and carotid intimal-medial thickness in children with insulin-dependent diabetes mellitus. J Am Coll Cardiol 2003;41:661-5.

33. Shroff RC, Donald AE, Hiorns MP, et al. Mineral metabolism and vascular damage in children on dialysis. J Am Soc Nephrol 2007;18:2996-3003.

34. Kavey RE, Allada V, Daniels SR, et al. Cardiovascular risk reduction in high-risk pediatric patients: a scientific statement from the American Heart Association Expert Panel on Population and Prevention Science; the Councils on Cardiovascular Disease in the Young, Epidemiology and Prevention, Nutrition, Physical Activity and Metabolism, High Blood Pressure Research, Cardiovascular Nursing, and the Kidney in Heart Disease; and the Interdisciplinary Working Group on Quality of Care and Outcomes Research: endorsed by the American Academy of Pediatrics. Circulation 2006;114:2710-38.

35. Meyer AA, Kundt G, Lenschow U, et al. Improvement of early vascular changes and cardiovascular risk factors in obese children after a six-month exercise program. J Am Coll Cardiol 2006;48:1865-70.

36. Wiegman A, Hutten BA, de Groot E, et al. Efficacy and safety of statin therapy in children with familial hypercholesterolemia: a randomized controlled trial. JAMA 2004;292:331-7.

37. Skilton MR, Viikari JS, Juonala M, et al. Fetal growth and preterm birth influence cardiovascular risk factors and arterial health in young adults: the Cardiovascular Risk in Young Finns Study. Arterioscler Thromb Vasc Biol 2011;31:2975-81.

38. Gale CR, Jiang B, Robinson SM, et al. Maternal diet during pregnancy and carotid intima-media thickness in children. Arterioscler Thromb Vasc Biol 2006;26:1877-82.

39. Dalla Pozza R, Ehringer-Schetitska D, Fritsch P, et al. Intima media thickness measurement in children: a statement from the Association for European Paediatric Cardiology (AEPC) Working Group on Cardiovascular Prevention endorsed by the Association for European Paediatric Cardiology. Atherosclerosis 2015;238:380-7.

40. Ben-Shlomo Y, Kuh D. A life course approach to chronic disease epidemiology: conceptual models, empirical challenges and interdisciplinary perspectives. Int J Epidemiol 2002;31:285-93.

41. Bots ML, Evans GW, Riley WA, et al. Carotid intima-media thickness measurements in intervention studies: design options, progression rates, and sample size considerations: a point of view. Stroke 2003;34:2985-94.
42. Urbina EM, Williams RV, Alpert BS, et al. Noninvasive assessment of subclinical atherosclerosis in children and adolescents: recommendations for standard assessment for clinical research: a scientific statement from the American Heart Association. Hypertension 2009;54:919-50.

43. Potter K, Reed CJ, Green DJ, et al. Ultrasound settings significantly alter arterial lumen and wall thickness measurements. Cardiovasc Ultrasound 2008;6:6:6.

44. de Groot E, Hovingh GK, Wiegman A, et al. Measurement of arterial wall thickness as a surrogate marker for atherosclerosis. Circulation 2004;109(1):III-33--30.

45. Mivelaz Y, Di Bernardo S, Boulos Ksontini T, et al. Feasibility and reliability of carotid intima-media thickness measurements in nonsedated infants. J Hypertens 2016;34:2227-32.

46. Liberati A, Altman DG, Tetzlaff J, et al. The PRISMA statement for reporting systematic reviews and meta-analyses of studies that evaluate healthcare interventions: explanation and elaboration. BMJ 2009;339:b2700.

47. Moher D, Shamseer L, Clarke M, et al. Preferred reporting items for systematic review and meta-analysis protocols (PRISMA-P) 2015 statement. Syst Rev 2015;4:1.

48. Shamseer L, Moher D, Clarke M, et al. Preferred reporting items for systematic review and meta-analysis protocols (PRISMA-P) 2015: elaboration and explanation. BMJ 2015;349:g7647.

49. Stroup DF, Berlin JA, Morton SC, et al. Meta-analysis of observational studies in epidemiology: a proposal for reporting. Meta-analysis Of Observational Studies in Epidemiology (MOOSE) group. JAMA 2000;283:2008-12.

50. Higgins JPT, Green S. Cochrane Handbook for Systematic Reviews of Interventions Version 5.1.0 [updated March 2011] The Cochrane Collaboration. 2011. www.handbook-5-1.cochrane.org.

51. Leyvraz M, Taffé P, Chatelan A, et al. Sodium intake and blood pressure in children and adolescents: protocol for a systematic review and meta-analysis. BMJ Open 2016;6:e012518.

52. Roulet $\mathrm{C}$, Bovet $\mathrm{P}$, Brauchli $\mathrm{T}$, et al. Secular trends in blood pressure in children: A systematic review. J Clin Hypertens 2017;19:488-97.

53. Santschi V, Chiolero A, Burnand B, et al. Impact of pharmacist care in the management of cardiovascular disease risk factors: a systematic review and meta-analysis of randomized trials. Arch Intern Med 2011;171:1441-53.

54. Santschi V, Chiolero A, Colosimo AL, et al. Improving blood pressure control through pharmacist interventions: a meta-analysis of randomized controlled trials. J Am Heart Assoc 2014;3:e000718.

55. Wells G, Shea B, O'Connell D, et al. The Newcastle-Ottawa Scale (NOS) for assessing the quality of nonrandomised studies in metaanalyses. http://www.ohri.ca/programs/clinical_epidemiology/oxford. asp (accessed Aug 2017).

56. Touboul PJ, Hennerici MG, Meairs S, et al. Mannheim carotid intima-media thickness and plaque consensus (2004-2006-2011). An update on behalf of the advisory board of the 3rd, 4th and 5th watching the risk symposia, at the 13th, 15th and 20th European Stroke Conferences, Mannheim, Germany, 2004, Brussels, Belgium, 2006, and Hamburg, Germany, 2011. Cerebrovasc Dis 2012;34:290-6.

57. Tang R, Hennig M, Bond MG, et al. Quality control of B-mode ultrasonic measurement of carotid artery intima-media thickness: the European Lacidipine Study on Atherosclerosis. J Hypertens 2005;23:1047-54.

58. Riley RD, Higgins JP, Deeks JJ. Interpretation of random effects meta-analyses. BMJ 2011;342:d549.

59. Egger M, Davey Smith G, Schneider M, et al. Bias in meta-analysis detected by a simple, graphical test. BMJ 1997;315:629-34

60. Schünemann H, Brożek J, Guyatt G, et al. Handbook for grading the quality of evidence and the strength of recommendations using the GRADE approach. Updated Oct 2013. http://gdt. guidelinedevelopment.org/app/handbook/handbook.html (accessed Aug 2017). 